\title{
Anisotropic light transmission of aligned carbon nanotube sheets coated substrates
}

\author{
MD Asiqur Rahman ${ }^{1}$, Ji Hyun Park ${ }^{1}$, Kieu Truong ${ }^{2}$, Dongseok Suh ${ }^{2}$, Giusy Scalia ${ }^{1}$ \\ ${ }^{I}$ Physics and Material Science Unit, University of Luxembourg, Luxembourg \\ ${ }^{2}$ Department of Energy Science, Sungkyunkwan University, Suwon 440-746, Korea
}

Received February 20, 2017; accepted March 29, 2017; published March 31, 2017

\begin{abstract}
We have performed optical investigations of layers formed by sheets of aligned multi-wall carbon nanotubes (MWCNTs). Nanotubes have extraordinary mechanical, electrical and optical properties when they are unidirectionally oriented. Free-standing CNT sheets drawn mechanically from vertically-grown MWCNT forests can be deposited on glass substrates, with nanotube bundles mostly oriented in the drawing direction. These CNT sheet layers have optical anisotropy, which can be detectable even for a single CNT sheet. We have studied the optical anisotropy in transmission, finding a similar value at different length scales indicating similarities in the orientational order of the nanotubes, thus preservation of their average degree of order. Finally, light transmission was observed when the samples were at $45 \mathrm{deg}$ between crossed polarizers.
\end{abstract}

The great interest in carbon nanotubes (CNTs) triggered by their discovery by Iijima [1] has led to significant scientific, engineering, and medical research for revealing the properties of these materials and ways to apply them. Carbon nanotubes are long cylinders from perfect honeycomb lattice walls of covalently bonded carbon atoms. Multi-wall carbon nanotubes (MWNTs), consist of two or more concentric cylindrical shells of graphene sheets coaxially arranged around a central hollow core. CNTs have extraordinary electrical and mechanical properties. MWNTs and single-wall CNTs are flexible and resilient [2] hollow tubular structures. The tubes that we have used here have diameters of about 20nm and lengths of 200300 micrometers [3]. The extraordinarily high anisotropy is not limited to the shape of the tubes but it is also in their properties and, in particular, in the absorption of light.

Due to their highly anisotropic optical characteristics, sheets formed by aligned CNTs are promising as linear optical polarizers. Lei Ren et al. reported a highly aligned SWCNT film of a thickness of 2 micrometers, deposited on a sapphire substrate, transparent to terahertz $(\mathrm{THz})$ radiation polarized perpendicular to the nanotube axis with an extinction ratio of about 10dB [4]. Later, Kyoung et al., by winding MWCNT sheets on a U-shaped polyethylene frame, obtained free-standing CNT layers of 9 micrometer thickness with an extinction ratio $\sim 37 \mathrm{~dB}$ in a spectral region from $0.1-2 \mathrm{THz}$ [5].

Herein we explore the optical properties of highly aligned MWNT sheets on glass substrate in the visible

http://www.photonics.pl/PLP wavelength range finding a clear anisotropy in the optical transmission as an effect of the CNT alignment.

Since, CNT sheets are attractive transparent electrodes due to their electrical conductivity and optical transparency, the study of their anisotropic properties and the dependence on the structural organization is of great importance for developing optical devices such as liquid crystal displays. In particular, the quality of the alignment of CNT strands is expected to be relevant for the properties of the sheets since it determines the anisotropy in the characteristics and, as consequence, the performance of the layers in optical devices or as a templating system.

We studied sheets of CNTs formed by one layer of about $100 \mathrm{~nm}$ in thickness. This value has to be considered as a simple indication of the order of magnitude of the thickness since the bundles of CNTs have, in general, a different diameter. Also, the CNTs are deposited as arrays and are not covering the complete surface, which is advantageous for enhancing the optical transmission and for a templating effect. However, the arrays present disuniformities in the surface coverage but, despite inhomogeneities in thickness and surface density, the CNT samples have reproducible optical features and provide similar results as discussed here.

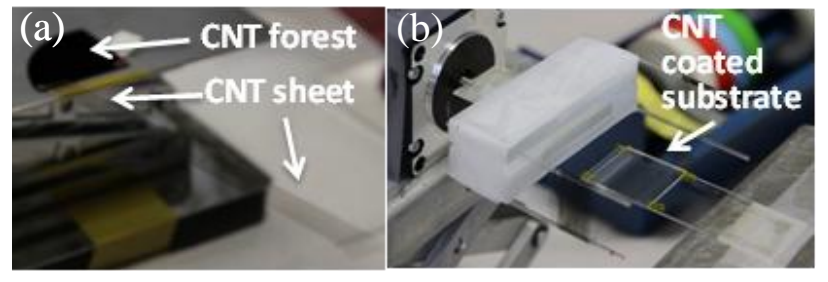

Fig. 1. Deposition of CNT sheet on glass substrate from a CNT forest.

The samples were prepared following the method introduced by Zhang et al. [6], built upon Jiang's innovative approach [7], pulling sheets from MWCNT forests. The basic properties of our sample were reported elsewhere [[2]]. CNT sheets are pulled out from a side wall of a forest and fixed to glass capillaries connected to a manually rotatable stage. As the stage was rotated, tubes can be continuously pulled out forming suspended stripes 
of connected CNTs, all aligned into the drawing direction, as shown in Fig. 1(a), which are then transferred to the glass substrate [Fig. 1(b)]. Because CNT sheets do not stick very well to the substrate surface, the adhesion between CNT and substrate was improved by using volatile organic solvents such as ethanol. Even after the solvent treatment the unidirectional alignment of CNTs holds. The nanotube orientation is evident in the optical microscope image in Fig. 2(a). In the right image (Fig. 2 (b)) we report the sketch of the CNT samples with the relevant directions of the nanotube layers (that means maximum absorption and maximum transmission) and with the directions of the input light and of its polarization.
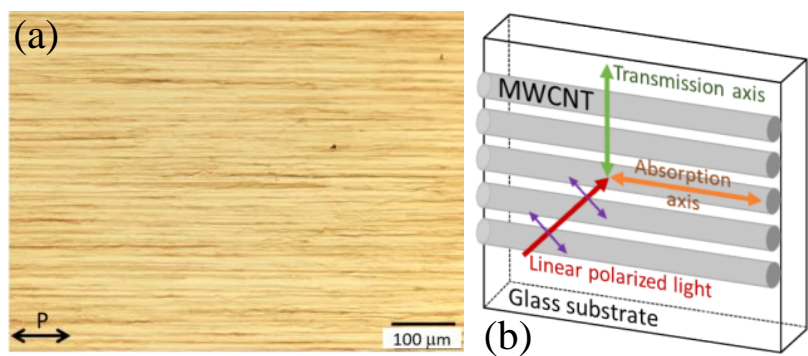

Fig. 2. a) Optical microscope image of a single sheet of aligned CNTs; b) sketch of the CNT sheets and their physical functions.

The optical study was performed using a polarizing optical microscope (Olympus BX51) equipped with a UV-VIS spectrophotometer (Avantes) for acquiring spectra of areas of characteristics known from optical images. A rotatable stage, between the polarizers, was used to study the angular dependence of the optical properties of the samples. To check the optical anisotropy we used also a standard UV spectrophotometer (Ultrospec 2000 pro), later repeating the same type of measurements with the spectrophotometer Avantes in order to compare the optical spectra from areas of different dimension. In fact, the standard spectrometer that we used probes areas in the order of $0.5 \mathrm{~cm}^{2}$. Instead, the spectrometer connected to the microscope analyzes much smaller parts of dimensions dependent on the chosen objective magnification, in our case an area of $0.38 \mathrm{~mm}^{2}$. In both cases an input polarizer was used to get a linearly polarized beam incident on the sample. The sample was then mounted with the CNT alignment direction as shown in Fig. 2(b), parallel or perpendicular to the polarizer transmission axis. Absorption and transmission spectra were measured in both configurations. In the upper part of Fig. 3, we have sketched the two basic configurations used for the transmission measurements reported in Fig. 3 (b) and (c). In Figure 3(b) the spectra are from the standard UV-Vis spectrometer while in the (c) image similar features were acquired with the system connected to the optical microscope. The transmittance for the aligned CNT sheet was measured between 400nm and

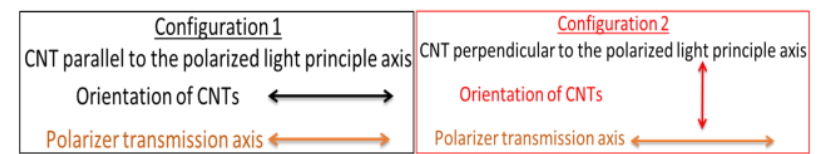

(a)
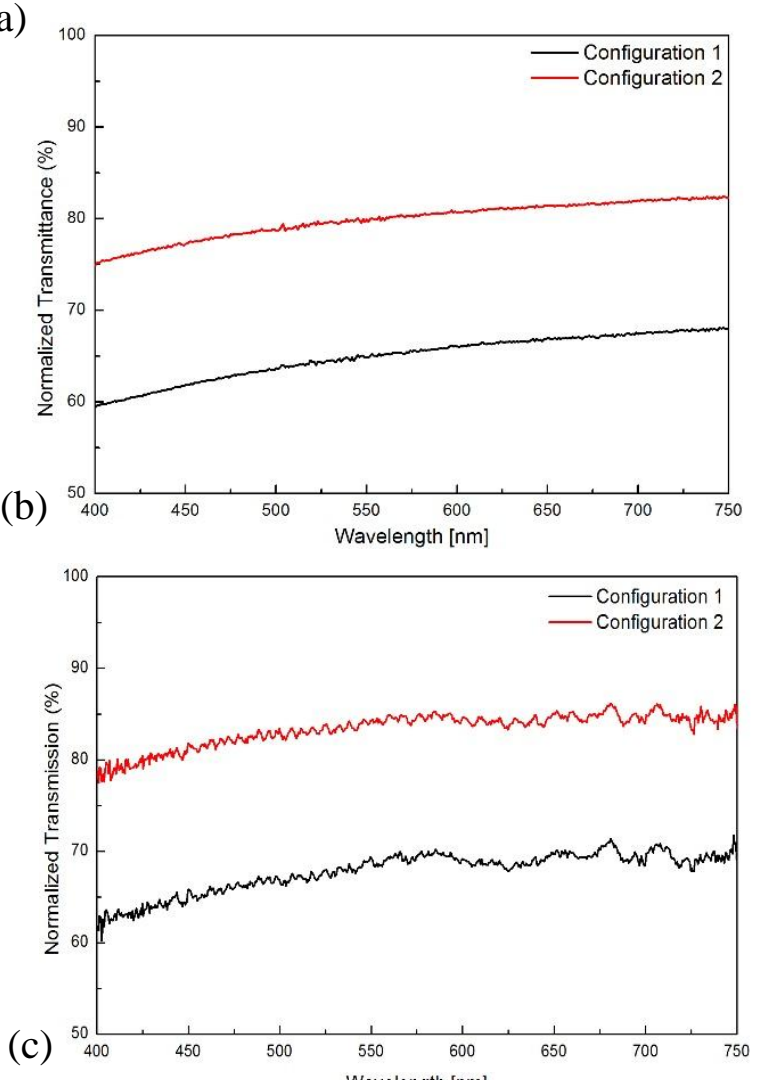

Fig. 3. a) Sketch of the alignment configurations used for the absorption measurements with b) a standard and c) local spectrophotometer. The red and black colors are associated to the configurations as in (a).

$750 \mathrm{~nm}$ obtaining values $\sim 75-82 \%$ for perpendicular polarization and $\sim 60-68 \%$ for parallel polarization. This indicates high transparency of single layer CNT sheets for nanotube orientation direction perpendicular to the polarization of the incident beam.

The transmission is clearly lower when the CNTs are aligned parallel to the incident polarization since this is the direction of maximum absorption. Thus this is the absorption axis of the film. An interesting aspect for optical applications is that the optical transmission is almost featureless, not showing deeps associated to absorption for particular wavelengths in the visible. The anisotropy in transmission is approximately equal to $15 \%$ in both systems, i.d. for the different spanned areas. The magnitude of the optical anisotropy is also constant for all the wavelengths in the visible range. Moreover, the value of the anisotropy in absorption is similar in the two cases, indicating that the properties can be regarded fairly similar at the two length scales, even despite the existing disuniformities in thickness and density and misalignment. 
The presence of misaligned bundles is especially visible using Atomic Force Microscopy (AFM) on a CNT sample, coated with an inorganic layer, at a smaller scale compared to the sizes analyzed optically. The left image shows the topography while the right one is taken in amplitude mode. We can notice that the tube bundles are not always straight showing curvatures and also that some tubes are positioned diagonally. However, the unidirectional alignment of CNTs is still well observable as shown in Fig. 4(a) and even clearer in Fig. 4(b).

Despite the presence of tubes not perfectly unidirectionally aligned, the fact that the optical properties are similar in regions of different length scale suggests that there the degree of orientational order of the CNT strands averages out at the same value.

Finally, we observed a change in transmission when the CNT samples were rotated from 0 to 90 degree in the optical microscope between crossed polarizers. At orientations of the transmission axis of the CNT sheets parallel and perpendicular with respect to the transmission axis of the polarizers, no transmission was observed. However, a maximum in intensity was found when CNT rotated at about 45 degree as shown in Fig. 5. We believe that this change in transmission is likely due to the anisotropic absorption, discussed previously.

In summary, we have studied the optical transmission of CNT sheets along the transmission and absorption directions. Despite being a very thin layer with some orientational misalignment, an anisotropic response in transmission is clearly detectable. In addition, the optical features are quite reproducible indicating that the degree of order is fairly similar in all the samples and, in the CNT sheet, similar for different length scales. Therefore, layers formed by aligned CNT sheets are attractive anisotropic elements for integration in optical devices.

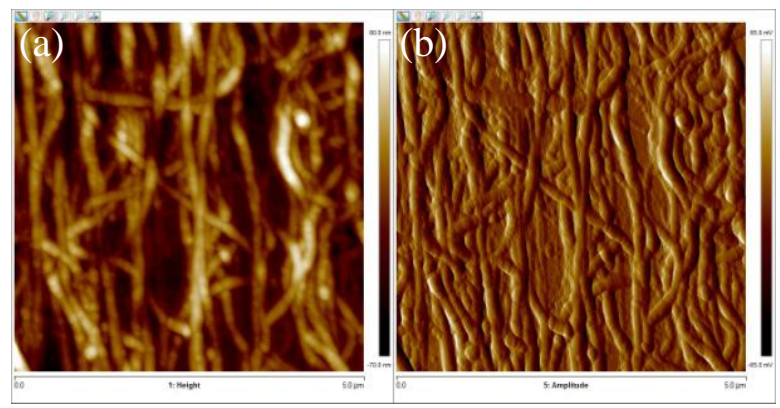

Fig. 4. AFM scan of CNT sheets in a) height and b) amplitude. The scan size is $5 \times 5 \mu \mathrm{m}^{2}$.

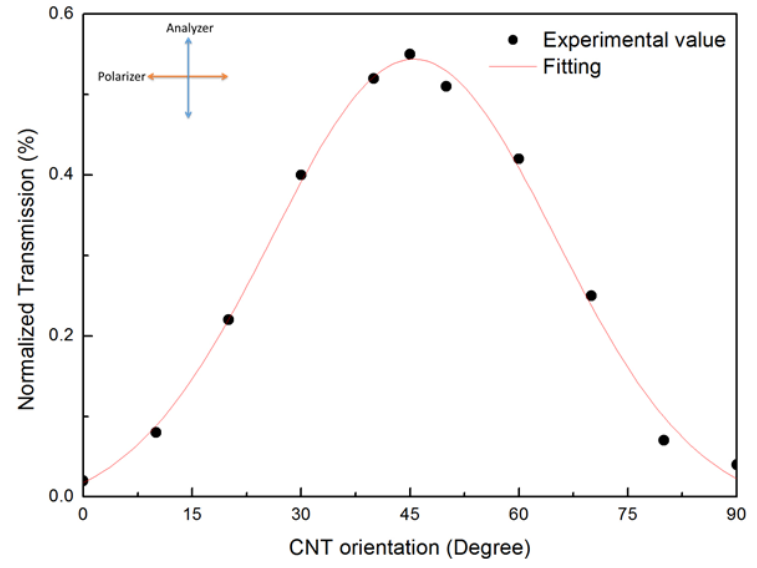

Fig. 5. Change in transmission between crossed polarizers of CNT sheets as the nanotube orientation direction is rotated.

This research was supported by Milicana C15/MS/10465407-R-AGR- 0654-10 from FNR (Luxembourg) and by the National Research Foundation of Korea (NRF-2016R1A2B2012336), funded by the Ministry of Science, ICT \& Future Planning (Republic of Korea). COST action IC 1208 is also acknowledged.

\section{References}

[1] S. Iijima, Nature 354, 56 (1991).

[2] S. Iijima, J. Chem. Phys. 104, 2089 (1996).

[3] T.K. Truong et al., Curr. Appl. Phys. 16, 1250 (2016).

[4] L. Ren et al., Nano Lett. 9, 7 (2009).

[5] J. Kyoung et al., Nano Lett. 11, 10 (2011).

[6] M. Zhang et al., Science 309, 5738 (2005).

[7] K. Jiang, Q. Li, S. Fan, Nature 419, 801 (2002). 